Ann. Génét. Sél. anim., 1977, 9 (1), 33-42.

\title{
Estimation de la valeur génétique des reproducteurs par la méthode de Henderson ( $\left.{ }^{1}\right)$
}

\author{
J. CHESNAIS \\ Département de Génétique animale, \\ Centr'e national de Recherches zootechniques, I.N.R.A., \\ 78350 Jouy-en-Josas
}

\section{Résumé}

Le but de cette note est d'exposer de façon simple les principes de base de la méthode de HENDERSON (BLUP). L'expression générale du BLUP est établie à partir de la notion d'information disponible (a priori et a posteriori) et des propriétés désirées pour les estimateurs. Trojs autres méthodes conduisant aux mêmes résultats que le BLUP sont indiquées ici. Finalement, on compare la méthode classique de comparaison aux contemporaines et la méthode d'Henderson dans le cas de l'estimation de la valeur génétique des taureaux laitiers.

\section{Introduction}

Sous l'effet des progrès réalisés au cours des dernières décennies (généralisation de l'I.A. dans certaines espèces, emploi de méthodes et de schémas de sélection efficaces, transfert de matériel génétique d'une région ou d'un pays à l'autre), plusieurs hypothèses inhérentes aux méthodes classiques d'évaluation des reproducteurs ont été invalidées.

De nouvelles méthodes, mieux adaptées aux circonstances actuelles, sont apparues.

Notre but iest ici de faire mieux connaître l'ưné d'entre elles, la méthode d'Henderson, en exposant de façon simple son principe général. Cette méthode est particulièrement intéressante parce qu'elle correspond au souci de préciser les hypothèses formulées pour l'évaluation des reproducteurs, hypothèses qui n’étaient souvent pas claires lors de l'emploi des méthodes classiques.

Pour rendre l'exposé plus concret, nous nous référerons fréquemment au cas des bovins laitiers, bien que le cadre d'application de la méthode d'HENDERSON soit beaucoup plus vaste.

(') Cet article a fait l'objet d'une communication lors du séminaire sur " I,'estimation de la valeur génétique des reproducteưts ", organisé à Toulouse les 6 et 7 octobre 1976 , par le Département de Génétiquue Animale de t'I:N.R.A. (France). 
Si le lecteur le désire, il pourra trouver des indications plus complètes sur cette méthode dans les articles publiés par son auteur (HENDERSON I963, I966, I972, I974).

\section{I. - Information disponible}

Pour l'évaluation des reproducteurs, nous disposons généralement de deux sources d'information :

-- les productions des animaux apparentés (information a posteriori);

- la connaissance du fait qu'un reproducteur provient d'une population déterminée (information a priori).

Illustrons ce point par un exemple simple. Soient deux taureaux - l'un tiré au hasard de la population Frisonne Pie-Noire, l'autre de la population Charolaise. Comment les comparer du point de vue de la production laitière?

- si nous ne disposons d'aucunes performances pour ces taureaux, le taureau Frison sera considéré par la plupart comme supérieur au taureau Charolais;

- si ces taureaux ont chacun une fille et que ces filles produisent la même quantité de lait, beaucoup considéreront encore le taureau Frison comme supérieur au taureau Charolais;

- si enfin ces taureaux ont chacun 200 filles dont les productions laitières sont en moyenne identiques, peu de gens considéreront ce taureau Frison comme supérieur à $c e$ taureau Charolais.

Cet exemple est extrême mais montre bien la dualité de l'information utilisée. On remarque aussi que l'information a priori a d'autant moins de poids que l'information a posteriori - les performances des anima'ux apparentés — est plus importante.

De ce point de vue nous pourrions distinguer deux types de méthodes (sans parler des méthodes totalement a priori) :

\section{a. - Les méthodes ne tenant compte que de l'information a posteriori}

Ce serait le cas d'une méthode de moindres carrés appliquée à un modèle où l'effet " reproducteur " est fixé. Une telle méthode conviendrait parfaitement dans l'une ou l'autre des situations suivantes :

- le nombre de données pour chaque reproducteur est tel que sa valeur génétique peut être estimée avec une précision suffisante;

- on ne possède aucune idée a priori sur la structure de la population des reproducteurs.

\section{b. - Les méthodes qui combinent les deux types d'information}

Dans le cas des populations d'animaux domestiques, on a souvent quelques idées sur la distribution des génotypes. D'autre part, pour certains reproducteurs, l'information issue des productions d'animaux apparentés est très réduite. On est 
donc amené à utiliser une méthode combinant l'information a priori et a posteriori.

Le processus d'échantillonnage des génotypes des reproducteurs est alors supposé connu. Ceux-ci sont considérés comme tirés au hasard d'une ou de plusieurs sous-populations, qui sont déterminées en fonction de connaissances a priori sur la structure générale de la population.

En conséquence, le génotype de chaque reproducteur est considéré comme aléatoire avec pour moyenne celle de la sous-population auquel il appartient et pour variance la variance génétique intra-population soit :

$$
g_{i} \sim \mathrm{L}\left(\mu_{i}, \sigma_{s}^{2}\right)
$$

Parmi les facteurs prévalant au choix de plusieurs sous-populations de taureaux laitiers on trouve notamment :

- plusieurs souches de taureaux peuvent parfois être distinguées dans la population (exemple en Pie-Noire : Holstein, Frison Français, Nord Hollande, etc...);

- par suite de la sélection, il est probable que les jeunes taureaux et les taureaux âgés appartiennent à des populations de niveaux génétiques différents;

- les taureaux utilisés en insémination artificielle sont sélectionnés différemment de ceux utilisés en monte naturelle;

- différents efforts de sélection peuvent aboutir à des différences régionales dans le niveau génétique moyen des reproducteurs.

Si le choix des sous-populations ou " groupes " ne correspond pas au processus réel d'échantillonnage des reproducteurs, les résultats sont erronés, puisque les hypothèses de départ sont inadéquates. Les estimées qui sont affectées le plus sont alors celles des animaux pour lesquels on possède peu de données, puisque ces estimées sont basées essentiellement sur l'appartenance de ces animaux à des souspopulations déterminées.

En résumé, de façon à tenir compte simultanément de l'information a priori et a posteriori, on est amené à considérer la valeur génétique des reproducteurs à évaluer comme aléatoire. On cherche alors un prédicteur de cette valeur connaissant les performances des animaux apparentés.

\section{II. - Choix d'un prédicteur}

Le problème consiste à trouver un prédicteur de la valeur génétique $g_{i}$ d'un reproducteur à partir des productions y d'animaux apparentés, sachant que :

$$
\begin{aligned}
g_{i} & \sim \mathrm{L}\left(\mu_{i}, \sigma_{s}^{2}\right) \\
\underline{y} & \sim \mathrm{L}(\underline{\theta}, \mathrm{V})
\end{aligned}
$$

où

$\mu_{:}=$moyenne de la population dont est 1 ssu le reproducteur considéré.

$\sigma_{s}^{2}=$ variance génétique intra population.

$\underline{\theta}=$ vecteur des espérances de $y$.

$\mathrm{V}=$ matrice de varianc $\_$-covariance de $y$. 
r. - Un critère logique pour le choix d'un prédicteur est qu'il minimise l'espérance de la somme des carrés des écarts à la vraie valeur, $\mathrm{E}\left(g_{i}-g_{i}\right)^{2}$.

On montre alors mathématiquement que le prédicteur vérifiant cette propriété est :

$$
\widehat{g_{i}}=\mathrm{E}\left(g_{i} / y\right)
$$

2. - En l'absence d'information sur la nature exacte de la distribution conjointe de $g_{i}$ et $y$, on se restreint à utiliser comme prédicteur de $g_{i}$ une fonction linéaire de $\underline{y}$, ce prédicteur s'écrit :

$$
\widehat{g}_{i}=\mu_{i}+\underline{c}^{\prime} i V^{-1}(\underline{y}-\underline{\theta})
$$

où $c_{i}=\operatorname{cov}\left(g_{i}, y\right)$, le vecteur des covariances entre $g_{i}$ et $y$.

Cette expression est plus générale que celle d'un index de sélection classique dans la mesure où $\mu_{i}$ peut varier d'un reproducteur à l'autre, s'ils sont issus de populations de moyennes différentes.

3. - Jusque-là nous avons supposé que les premiers et les seconds moments de la distribution, c'est-à-dire les moyennes, variances et covariances $\left(\mu_{i}, \theta, \sigma_{s}^{2}\right.$, $\left.c_{i}, \mathrm{~V}\right)$ étaient connues sans erreur. Celà n'est en fait jamais le cas.

Cependant, on dispose généralement de nombreuses estimées sur les variances et. covariances des variables considérées, et l'expérience prouve que ces estimées sont relativement stables au cours du temps et pour des milieux différents. On peut donc, en première approximation, les considérer comme connues (une deuxième étape consisterait d'une part à étudier l'effet des erreurs d'estimation des variances et covariances sur la précision des résultats, d'autre part à mettre au point des méthodes permettant d'évaluer directement ces paramètres...).

A l'inverse, il est peu justifié de considérer comme connues les moyennes des'variables $g_{i}$ et $y$, dans la mesure où l'on ne possède pas d'estimées préalables pour ces moyennes. Il est donc nécessaire de les estimer en même temps que la valeur génétique des reproducteurs. Ce qui est fait en imposant la condition supplémentaire :

$$
\mathrm{E}\left(\widehat{g_{i}}\right)=\mathrm{E}\left(g_{i}\right)
$$

c'est-à-dire que l'espérance du prédicteur doit égaler celle de la valeur à prédire. Le prédicteur recherché s'écrit alors :

$$
g_{i}=\widehat{\mu_{i}}+\underline{c}^{\prime} i \mathrm{~V}^{-1}(\underline{y}-\underline{\theta})
$$

où $\widehat{\mu_{i}}$ et $\widehat{\theta}$ sont des estimations de $\mu$ et de $\theta$ obtenues par la méthode des moindres carrés généralisés.

En effet, si l'on exprime $\mu_{i}$ et $\theta$ comme :

$$
\underline{\mu_{i}}=\underline{p_{i}^{\prime} i b} \quad \text { et } \quad \underline{\theta}=\mathrm{X} \underline{b}
$$

où le vecteur $p_{i}$ et la matrice $\mathrm{X}$ décrivent l'incidence des effets à estimer $b$ sur les moyennes, on trouve d'après les critères fixés précédemment que :

$$
\widehat{b}=\left(\mathrm{X}^{\prime} \mathrm{V}^{-1} \mathrm{X}\right)^{-} \quad \mathrm{X}^{\prime} \mathrm{V}^{-1} \underline{y}
$$


Les effets $b$ sont définis en fonction des hypothèses. Dans le cas de l'évaluation des taureauxx laitiers, ce sont souvent des effets propres au troupeau, à l'année, à la saison, et à la moyenne génétique de la population dont est issu le taureau considéré.

La généralisation de l'expression (3) à l'ensemble des génotypes de la population s'écrit :

$$
\bar{g}=\underline{\mu}+C^{\prime} V^{-1}(\underline{y}-\underline{\hat{H}})
$$

où

$\dddot{g}=$ vecteur des estimations des valeurs génétiques des reproducteurs

$\underline{\mu}=$ vecteur des estimations des espérances de $\underline{g}$

$\widehat{\theta}=$ vecteur des estimations des espérances des observations

$\overline{\mathrm{C}}=$ matrice de covariance de $g$ et $y$

$\mathrm{V}=$ matrice de variance-covariance des observations.

En exprimant les estimations des moyennes en fonction des paramètres du modèle on obtient :

$$
\underline{\bar{g}}=\mathrm{P} \hat{b}+\mathrm{C}^{\prime} \mathrm{V}^{-1}(\underline{y}-\mathrm{X} \underline{b})
$$

Pour résumer, en substituant aux moyennes leurs estimées de moindres carrés généralisés, on obtient comme prédicteur de $g$ une fonction linéaire des observations qui :

-- tient compte de l'information disponible sur l'échantillonnage des reproducteurs considérés;

- a pour espérance l'espérance de la valeur à prédire (dite " non biaisé ");

- minimise l'espérance de la somme des carrés des erreurs de prédiction.

Un tel prédicteur a été nommé BLUP (Best Linear Unbiased Prediction) par HENDERSON. On peut montrer que dans l'ensemble des prédicteurs linéaires " centrés ", le BLLP est celui qui maximise la corrélation entre le prédicteur et la valeur à prédire.

\section{III. - Autres méthodes permettant d'arriver au Blup}

Le prédicteur décrit précédemment peut en fait être obtenu par différentes méthodes.

Certaines de ces méthodes sont intéressantes parce que faciles à appliquer, d'autres parce qu'elles apportent un éclairage supplémentaire sur la signification du BLUP.

\section{I. - Modèle mixte d'HENDERSON}

Un des inconvénients majeurs de l'approche indiquée ci-dessus (dite des index de sélection modifiés) est qu'elle requiert l'estimation des moyennes de $g$ et $y$ par la méthode des moindres carrés généralisés. Ceci nécessite l'inversion de la 
matrice de variance-covariance $V$ des observations, qui en général n'est pas diagonale. Il s'en suit que la méthode n'est applicable telle quelle qu'à un nombre limité d'observations (de l'ordre de 500 avec les moyens actuels de calcul) alors que par exemple l'évaluation des taureaux laitiers met en jeu des milliers sinon des millions de données.

Cependant, Henderson a montré l'équivalence du BLUP et des solutions du " modèle mixte ".

Soit le modèle :

$$
\underline{y}=\underline{\mathrm{X} b}+\underline{\mathrm{Z}} \underline{u}+\underline{e}
$$

où $y$ est le vecteur des observations

$\bar{u}$ est un vecteur de variables aléatoires non observables

$\vec{e}$ est le vecteur des variables résiduelles associées à chaque observation

$\mathrm{X}$ et $\mathrm{Z}$ décrivent l'incidence des inconnues $b$ et $u$ sur chaque observation (X et $Z$ sont généralement composées de o et de $\mathrm{I}$ ).

On suppose dans ce modèle que :

$$
\mathrm{E}\left[\begin{array}{c}
\frac{y}{u} \\
\frac{e}{e}
\end{array}\right]=\left[\begin{array}{c}
\mathrm{X} b \\
- \\
\mathrm{o} \\
\mathrm{o}
\end{array}\right] \text { et } \operatorname{Var}\left[\begin{array}{c}
\frac{y}{u} \\
\frac{e}{e}
\end{array}\right]=\left[\begin{array}{lll}
\mathrm{V} & \mathrm{ZG} & \mathrm{R} \\
\mathrm{GZ} & \mathrm{G} & \mathrm{o} \\
\mathrm{R} & \mathrm{o} & \mathrm{R}
\end{array}\right]
$$

les équations dites " du modèle mixte " sont alors les suivantes :

$$
\left[\begin{array}{ll}
\mathrm{X}^{\prime} \mathrm{R}^{-1} \mathrm{X} & \mathrm{X}^{\prime} \mathrm{R}^{-1} \mathrm{Z} \\
\mathrm{Z}^{\prime} \mathrm{R}^{-1} \mathrm{X} & \mathrm{Z}^{\prime} \mathrm{R}^{-1} \mathrm{Z}+\mathrm{G}^{-1}
\end{array}\right] \quad\left[\begin{array}{l}
b^{*} \\
u^{*}
\end{array}\right]=\left[\begin{array}{l}
\mathrm{X}^{\prime} \mathrm{R}^{-1} y \\
\mathrm{Z}^{\prime} \mathrm{R}^{-1} y
\end{array}\right]
$$

On montre mathématiquement que, par comparaison à l'expression (6), les solutions de ces équations sont telles que :

$$
\left.\underline{b^{*}}=\underline{b} \quad \text { et } \quad \underline{u}^{*}=\mathrm{C}^{\prime} \mathrm{V}^{-1} \underline{\underline{y}}-\mathrm{X} \underline{b}\right)
$$

Le BLUP s'écrit donc, en fonction des solutions du modèle mixte :

$$
\underline{g}=\underline{P}^{*}+\underline{u^{*}}
$$

Cette méthode suscite plusieurs remarques :

a. - Bien que le terme "modèle mixte " soit utilisé, il ne s'agit pas ici d'estimer simultanément des effets fixés et des variances d'effets aléatoires, comme ce pourrait être le cas pour un calcul d'héritabilité. Au contraire, la variance des éléments aléatoires du modèle est supposée connue. La signification de ce modèle est donc bien différente de celle du modèle mixte "classique ".

$b$. - Le système d'équations (7) est en général plus facile à résoudre que celui des équations de moindres carrés généralisés. En effet, dans la plupart des applications, la matrice $\mathrm{R}$ est diagonale et l'inverse de la matrice $\mathrm{G}$ se calcule aisément. Ces équations sont alors celles des moindres cairús dans l'hypothèse où les effets $u$ sont fixés, modifiées par l'addition des termes de la matrice $\mathrm{G}^{\mathbf{- 1}}$.

c. - En pratique, lorsque le nombre de paramètres à estimer et donc d'équations est trop élevé pour qu'on puisse résoudre le système directement par inver- 
sion de la matrice des coefficients, on élimine certaines de ces équations par substitution. Le système des équations restantes est alors résolu par itérations successives.

\section{2. - Méthode des moindres carrés régressés}

DEMPFLE (I976) a montré que la méthode des moindres carrés régressés donne les mêmes résultats que le BLUP lorsqu'elle est appliquée rigoureusement. Dans un premier temps, les valeurs génétiques des reproducteurs sont considérées comme fixées et estimées comme les autres effets du modèle par la méthode des moindres carrés. Les estimations obtenues se basent alors uniquement sur l'information provenant des performances d'animaux apparentés.

Dans un deuxième temps, les valeurs génétiques sont reconnues comme aléatoires et régressées sur leurs estimées de moindres carrés. Si l'on tient compte des variances et covariances entre estimées de moindres carrés, les prédicteurs obtenus ainsi sont ceux du BLUP.

\section{3. - Approche de type Bayésien}

DEMPFLE (1976) montre que le BLUP correspond à l'application de méthodes Bayesiennes à un modèle linéaire lorsque les lois des variables $g$ et $y$ sont normales. On peut même montrer que le BLUP correspond à l'utilisation conjointe de deux estimateurs indépendants de la même quantité, l'un a priori, l'autre a posteriori, chacun de ces estimateurs étant pondéré par la quantité d'information correspondante.

\section{IV. - Méthode classique de comparaison aux contemporaines et Blup}

Jusqu'à une époque récente, l'estimation de la valeur génétique des taureaux laitiers était principalement faite par la méthode de comparaison aux contemporaines. La valeur génétique d'un taureau est alors estimée à partir des performances $\underline{y}$ de ses filles par la formule :

$$
\widehat{g_{i}}=\operatorname{cov}\left(g_{i}, \underline{y}\right)[\operatorname{var} y]^{-1}(y-\underline{-} y)
$$

soit $\bar{g}_{i}=\underline{c}_{i}^{\prime} V^{-1}(\underline{y}-\bar{y})$

où chaque élément du vecteur $(y-\bar{y})$ représente l'écart entre la performance d'une fille du taureau $i$ et la moyenne des performances de ses contemporaines d'étable.

Si nous comparons cette formule à celle donnée pour le BLUP par l'expres$\operatorname{sion}(3)$

$$
\bar{g}_{i}=\widehat{\mu}_{i}+c_{-}^{\prime}{ }^{\prime} V^{-1}(\underline{y}-\widehat{\theta})
$$


Nous constatons les différences suivantes :

I. - Dans le cas de la méthode de comparaison aux contemporaines, $g_{i}$ est régressé par rapport à une seule moyenne, supposée connue et égale à o. Ceci suppose que les taureaux évalués sont issus d'une seule et même population. Or, comme nous l'avons vu au début de cet article, de nombreuses raisons font que cette

\section{TABLEAU I}

Valeur génétique moyenne des pères des contemporaines pour 3 taureaux de race holstein, dans un échantillon des troupeaux-années-saisons ou ils ont été utilisés. (D'après L. ScHAEFFER, I995). (Mean genetic value of contemponaries fathers for 3 Holstein bulls in a herd-year-season sample where they were used)

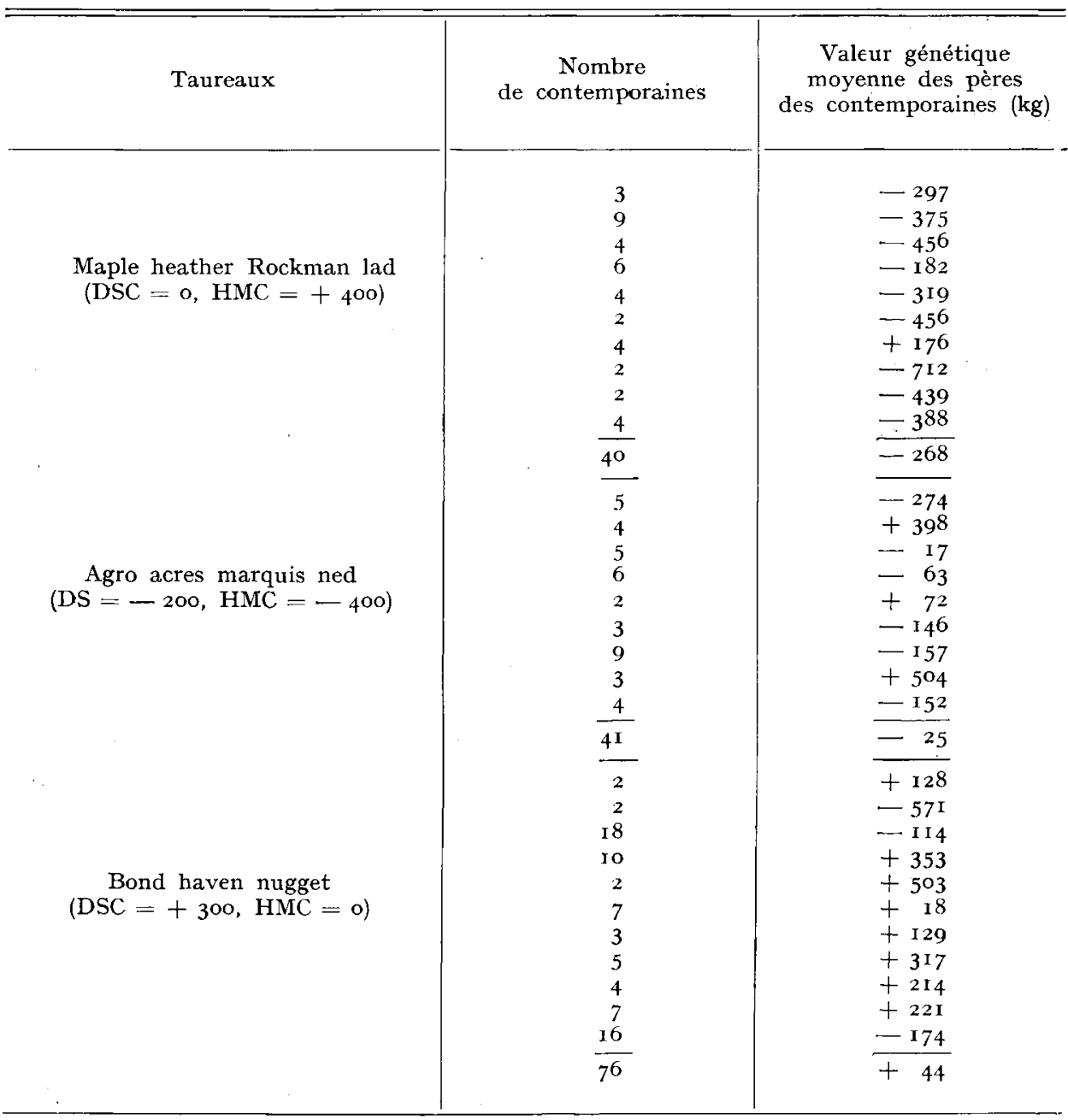
(BLUP).

*DSC $=$ Index (kg de lait) obtenu par la méthode de comparaison directe des taureaux $\mathrm{HMC}=$ Index (kg de lait) obtenu par la méthode de comparaison aux contempcraines. 
hypothèse est probablement fausse dans les conditions actuelles. Les estimations obtenues par la méthode de comparaison aux contemporaines sont alors biaisées surtout celles des taureaux pour lesquels on possède peu de données.

Le BLUP, au contraire, laisse le choix des hypothèses formulées sur l'échantillonnage des taureaux : une ou plusieurs populations (et donc une ou plusieurs moyennes $\mu_{i}$ ) sont distinguées suivant les hypothèses retenues.

2. - Avec la méthode de comparaison aux contemporaines, l'espérance de chaque performance est estimée par la moyenne des performances des contemporaines d'étable, et non, comme dans. le BLUP, par un estimateur de moindres carrés généralisés.

Cela revient à supposer que la valeur génétique moyenne des pères des contemporaines est la même dans chaque étable pour tous les taureaux évalués. Autrement dit, contrairement à ce qui se passe avec le BLUP, on ne tient pas compte des différences de compétition à laquelle sont exposés les taureaux.

Or il est clair que dans les conditions actuelles, de telles différences de compétition existent (intensités ou objectifs de sélection différents dans différentes régions, progrès génétique, utilisation préférentielle de certains taureaux dans certains types d'élevage, etc...).

Prenons l'exemple de 3 taureaux Canadiens. Dans le tableau I, nous avons reporté les index de ces taureaux calculés par le BLUP et par la méthode de comparaison aux contemporaines, ainsi que la valeur des pères des contemporaines de leurs filles dans un échantillon des troupeaux-années-saisons où ils ont été utilisés. On peut voir que les trois taureaux n'ont en moyenne pas été comparés à des taureaux de niveau génétique semblable.

Le biais qui en résulte dans la méthode de comparaison aux contemporaines est important, si l'on se réfère aux écarts entre les index calculés par les 2 méthodes. Notons que ce type de biais constitue la majeure faiblesse de la méthode de comparaison aux contemporaines. Tous les taureaux peuvent être affectés, quel que soit le nombre de filles sur lequel ils sont jugés, et les jeunes taureaux sont systématiquement défavorisés par rapport aux vieux en présence de progrès génétique.

\section{Conclusion}

Deux points importants se dégagent de cette étude :

- la théorie des index de sélection s'explique bien si l'on y introduit la notion d'information a priori;

- la méthode d'Henderson permet de généraliser la théorie des index de sélection au cas où l'on distingue plusieurs populations de reproducteurs et /ou au cas où les reproducteurs font face à différents niveaux de compétition.

Nous nous sommes limités ici à l'exposé du principe général du BLUP. Signalons également que :

- plusieurs méthodes tendent à donner des approximations du BLUP tout en réduisant le temps de calcul lorsque le nombre de données à analyser est très élevé. L'efficacité de c€s méthodes ne peut être mesurée que si les approximations faites sont clairement définies, ce qui est rarement le cas;

- le BLUP s'adapte bien aux raffinements apportés actuellement aux méthodes d'estimation de la valeur génétique des reproducteurs : correction des biais dus à la sélection des reproducteurs ou de leurs apparentés, calcul d'index sur dif- 
férents types d'animaux apparentés, sur des répétitions de performances ou sur plusieurs caractères (HENDERSON, I972, I974, I975, I976; SCHAEFFER et WILTON, I976).

Reçu poù publication en février 1977.

\section{Remerciements}

Nous remercions le $\mathrm{D}^{\mathrm{r}}$ Dempree (Lehrstahl für Tierzucht der Technischen Universitât München) pour ses utiles suggestions et remarques.

\section{Summary}

\section{Estimation of breeding values using Henderson's method}

An attempt is made here to present in a simple manner the basic philosophy underlying HeNDERSON's Best Linear Unbiased Prediction method (BLUP). The concept of a priori information is used to introduce the method. The general derivation of BLUP is given along with two other methods leading to the same estimates. Finally, a comparison is made between the contemporary comparison method and BLUP as used for dairy sire evaluation.

\section{Références bibliographiques}

DEMPFLE L., 1976. Comparison of several sire evaluation methods in dairy cattle breeding. Proc. of the $27^{\text {th }}$ meeting of the E.A.A.P., Zurich.

Dempfle L., 1976. 'Relation entre méthode bayésienne et BLUP. Ann. Génét. Sél. Anim., 10.

HENDERSON C. R., I963. Selection index and expected genetic advance. In statistical genetics and Plant Breeding. NAS-NRC, 982-I003.

HeNDERSON C. R., I966. A sive evaluation method which accounts for unknown genetic and environmental trends, herd differences, seasons, age effects and differential culling. Symp. on Estimating Breed. Values of Dairy Sires and Cows. USDA Mimeo.

Henderson C. R., 1972. Sire evaluation and genetic trends. Proc of the An. Breed. Genetics Symp., I972, Blacksburg, I0-4I.

Henderson C. R., I974. General flexibility of linear model techniques for sire evaluation. J. Dairy Sci., 57, 963-972.

Henderson C. R., 1975. Rapid method for computing the inverse of a relationship matrix. J. Dairy Sci., 58, 1727-1 730 .

HENDERSON C. R., I975. Unse of relationships among sires to increase accuracy of sire evaluation J. Dairy Sci., 58, I 73 I-I $73^{8}$.

Henderson C. R., 1975. Use of all relatives in intraherd prediction of breeding values and producing abilities. J. Dairy Sci., 58, 1910-1916.

HENDERSON C. R., 1975. Inverse of a matrix of relationships due to sires and maternal grandsires. J. Dairy Sci., 58, I9I 7-192 I.

Henderson C. R., 1975. Best linear unbiased estimation and prediction under a selection model. Biometrics, 31, 423:447.

Henderson C. R., I976. Multiple trait sire evaluation using the relationships matrix J. Dairy Sci., 59, $769-774$.

SCHAFFER L., 1975. Communication personnelle.

Schaeffer L. R., Wilton J. W., I976. Methods of sire evaluation for calving ease. J. Dairy Sci., 59, 544-55I. 